山्山FFRANÇAISE

$\supset \mathrm{DE}$

$\stackrel{1=1}{\simeq}$ PÉDAGOGIE

\section{Revue française de pédagogie}

Recherches en éducation

174 | janvier-mars 2011

Évaluation, développement professionnel et organisation scolaire

\title{
Les évaluations intermédiaires des élèves dans le cadre des cycles d'amélioration continue de l'enseignement
}

Intermediate assessment of students within the framework of the cycles of continuous improvement of teaching

Las evaluaciones intermediarias de los alumnos en el marco de los ciclos de mejora continua de la enseñanza

Die Zwischenbeurteilung der Schüler im Zyklus der ständigen Verbesserung des

Unterrichtswesens

Leslie Nabors Oláh

\section{OpenEdition}

Journals

Édition électronique

URL : http://journals.openedition.org/rfp/2833

DOI : $10.4000 /$ rfp.2833

ISSN : 2105-2913

Éditeur

ENS Éditions

Édition imprimée

Date de publication : 15 mars 2011

Pagination : $61-70$

ISBN : 978-2-7342-1203-4

ISSN : 0556-7807

\section{Référence électronique}

Leslie Nabors Oláh, «Les évaluations intermédiaires des élèves dans le cadre des cycles

d'amélioration continue de l'enseignement », Revue française de pédagogie [En ligne], 174 I janvier-mars 2011, mis en ligne le 15 mars 2015, consulté le 01 mai 2019. URL : http://journals.openedition.org/ rfp/2833; DOI : 10.4000/rfp.2833 


\section{Les évaluations intermédiaires des élèves dans le cadre des cycles $d^{\prime}$ amélioration continue de l'enseignement

\author{
Leslie Nabors Oláh
}

L'objectif de cet article est d'examiner l'usage des évaluations intermédiaires et des outils qui les accompagnent pour améliorer l'enseignement des mathématiques à l'école élémentaire aux États-Unis. Nous utilisons le terme d'« évaluations intermédiaires " pour caractériser des évaluations cycliques des connaissances et des compétences, limitées dans le temps, qui servent comme données agrégées pour établir des comparaisons entre les classes, les écoles, et à l'échelle du district. Nous étudions plus particulièrement comment ce système d'information et cette base de données changent les conditions d'instruction et l'usage pédagogique qu'en font les enseignants avec les élèves dans leur classe.

Descripteurs (TESE) : évaluation par les enseignants, évaluation formative, traitement des données, pratique pédagogique, processus d'apprentissage.

Aux États-Unis, la plupart des débats sur les évaluations intermédiaires portent sur les cycles d'amélioration continue, processus de retour d'informations visant à améliorer l'enseignement et les apprentissages des élèves. Les étapes successives d'évaluation, d'analyse et d'interprétation des résultats, puis de planification et de mise en œuvre de nouvelles pratiques pédagogiques constituent le déroulé logique de ce cycle. Cet article présente les résultats d'une étude de trois ans financée par la National Science Foundation, qui cherchait à connaître la façon dont les enseignants utilisent les résultats des évaluations intermédiaires dans le cadre du cycle d'amélioration continue de l'enseignement.

\section{LES CYCLES D'AMÉLIORATION CONTINUE DE L'ENSEIGNEMENT}

Les enseignants croulent sous une avalanche d'outils visant à évaluer les apprentissages des élèves. Ces outils sont créés par différents prestataires - entreprises privées, organisations à but non lucratif, éditeurs de manuels scolaires - ainsi que par les États et les districts scolaires (Burch, 2010). Naturellement, les enseignants créent aussi leurs propres tests, quiz et autres outils quotidiens d'évaluation des performances des élèves. Concrètement, à l'école élémentaire, un enseignant est amené à faire passer 
toutes les 4 à 6 semaines, pour chaque discipline d'enseignement, à la fois un test de fin de séquence conformément au programme scolaire, une évaluation intermédiaire imposée par le district et d'autres situations d'évaluation (devoirs à la maison, interrogations orales, quiz, etc.). De plus, les enseignants sont susceptibles d'évaluer les élèves dont l'anglais n'est pas la langue maternelle ou qui font l'objet d'un enseignement spécialisé. Sans surprise, ces évaluations nombreuses produisent une abondance de données, certaines sous la forme de résultats ou de pourcentages bruts, d'autres sous la forme de réponses semi-guidées ou d'une rédaction libre. Toutes ces données peuvent être utiles aux enseignements et aux apprentissages des élèves. Que ce soit au niveau de l'état, du district, de l'établissement ou de la classe, l'idée de base de cycles d'amélioration continue consiste à amener les professionnels à prendre des décisions ayant une influence sur les performances des élèves. Cette amélioration dépend de l'accès à des informations de qualité et de la capacité à les analyser et à agir en conséquence (Supovitz, 2006).

Ces processus d'amélioration continue ont été lancés par les États, les districts et des organismes externes de réforme. Le Northwest Regional Educational Laboratory (2001) divise par exemple le cycle d'amélioration continue en quatre étapes : comprendre, planifier, mettre en œuvre et réfléchir. La Technology Alliance prévoit quant à elle six étapes : définir les résultats souhaités, choisir les questions de recherche, recueillir et organiser les données, leur donner du sens, agir et évaluer les initiatives qui ont été prises. La Coalition of Essential Schools préconise elle aussi une division en six étapes : déterminer une vision de l'enseignement et de l'apprentissage, formuler des questions pouvant faire l'objet de recherches, préparer les cours, enseigner et recueillir les données, les analyser et en tirer les conséquences en termes de changement de pratiques pédagogiques (Cushman, 1999). En outre, d'innombrables districts scolaires diffusent des versions modifiées du cycle Deming (planifier, faire, étudier, agir), présenté comme un modèle d'amélioration continue. Cette logique ne vaut pas seulement pour les États-Unis, mais aussi pour la Nouvelle-Zélande où le ministère de l'Éducation a lancé un processus en quatre étapes : identifier les besoins d'apprentissage des élèves, des enseignants et de l'encadrement ; définir les questions d'enquête ou de recherche; mettre au point et faire des expériences sur ces questions ; évaluer leur effet sur différents publics (Timperley, Wilson, Barrar et al., 2007).
Aux États-Unis, ces cycles ont été appliqués à différents niveaux : dans les districts, les établissements et les classes. Au niveau des districts, les résultats aux tests standardisés ont permis de définir des objectifs de performance pour les districts et les établissements, de déterminer le type d'accompagnement nécessaire et la gamme de sanctions pour les établissements défaillants (Supovitz, 2006). Au niveau des établissements, les données issues des évaluations de l'État servent à identifier les besoins en développement professionnel, à évaluer les enseignants et à repérer les élèves en grande difficulté (Kerr, Marsh, Ikemoto et al., 2006). Les données d'évaluation intermédiaire sont utilisées aux mêmes fins, ainsi qu'à la promotion d'une pédagogie différenciée et au suivi des progrès des enseignants et des élèves utiles à l'accomplissement des objectifs de performance (Bulkley, Christman, Goertz et al., 2008 ; Clune \& White, 2008). Plus précisément, les recherches dans ce domaine portent principalement sur les différentes façons dont les enseignants suscitent l'intérêt des élèves et répondent en temps réel à leurs erreurs ou mauvaises interprétations (Coburn \& Talbert, 2006). Ce processus est plus connu sous le nom de « mettre au jour, interpréter et agir » (Bell \& Cowie, 2001) ou " mettre au jour, reconnaître et utiliser les informations 》 (Ruiz-Primo \& Furtak, 2004). Black et William (2006, p. 88) ont affiné ce cycle en six étapes consécutives :

- définir un objectif d'apprentissage sanctionné par une évaluation formative ;

- favoriser des échanges à partir des réponses des élèves ;

- effectuer le recueil et l'interprétation des réponses des élèves ;

- prendre des initiatives à partir de cette interprétation ;

- effectuer le recueil et l'interprétation de chaque initiative testée ;

- effectuer la transition vers l'objectif suivant.

Si les modèles d'amélioration continue de l'enseignement diffèrent en termes d'échelle d'action et de nombre d'étapes nécessaires, tous comportent trois éléments fondamentaux : le recueil d'informations, leur interprétation et une prise d'initiatives de la part de l'enseignant en fonction de cette interprétation. Nous présentons dans cet article les résultats d'une étude prospective de trois ans sur l'usage que font les enseignants des évaluations intermédiaires, dans le cadre de ces différents cycles. L'étude partait de la question suivante : "Quels enseignements les professeurs d'école élémentaire retirent-ils, individuellement et collectivement, des résultats de l'évaluation 
intermédiaire en mathématiques et qu'en font-ils pour transformer leur enseignement en termes de contenus, de pédagogie et de travail avec chaque élève ? "

\section{LE CURRICULUM ET LE CONTEXTE DES ÉVALUATIONS}

\section{Le cas du district de Philadelphie}

Dans ce district de 173000 élèves, les évaluations intermédiaires étudiées s'inscrivaient dans une réforme plus large visant à élever le niveau des élèves. Durant l'année scolaire 2002-2003, le district a mis en place un programme d'enseignement unique et a administré pour la première fois des évaluations aux grade 3 (équivalent du CE2) et grade 8 (équivalent de la $4^{\mathrm{e}}$ ) afin de donner aux enseignants des informations sur les acquis des élèves après 6 semaines de cours portant sur un même thème. Selon plusieurs responsables du district, ces évaluations devaient servir " d'outils d'enseignement ", c'est-à-dire d'outils d'accompagnement donnant aux enseignants des informations opportunes sur les apprentissages réels de leurs élèves. Une période de cinq semaines de cours était couronnée par une série d'évaluations, la sixième servant au bilan et/ou à l'approfondissement des matières testées. L'objectif affiché était d'évaluer, dans une période de temps limité, les connaissances et les compétences des élèves par rapport à un ensemble précis d'objectifs, afin d'éclairer les décisions pédagogiques prises en classe. Les résultats pouvaient être ensuite utilisés plus globalement à l'échelle de l'établissement et du district.

Bien que la période de six semaines chevauchât plusieurs chapitres de cours, l'évaluation intermédiaire ne visait qu'à tester les concepts et objectifs enseignés depuis la précédente évaluation. Tous les items de ces évaluations (20 items en grade 3 et 25 en grade 8) étaient rédigés sous forme de questions à choix multiples. Entre chaque période, le prestataire de tests privé (The Princeton Review) et les administrateurs du district scolaire de Philadelphie élaboraient ensemble les évaluations suivantes. Le district établissait une première ébauche de tests, à partir de laquelle The Princeton Review proposait une première série d'items. Cette base de travail donnait lieu ensuite à des corrections et des amendements successifs des deux institutions, jusqu'à ce que le district fût satisfait du produit final. Grâce à ce processus itératif, les tests étaient en phase de développement tout au long de l'année.

\section{Le cas du district de Cumberland}

Dans ce district de 7500 élèves, l'enseignement des mathématiques suit le rythme de progression fixé dans le guide officiel du district. Ce guide d'une page indique le nombre de leçons par chapitre, le nombre de jours nécessaires pour couvrir tout le chapitre et la date de fin prévue pour chaque chapitre. Les évaluations formelles des mathématiques dans l'enseignement primaire comportent des tests d'entraînement (intermédiaires), des tests de fin de chapitre (formatifs et/ou sommatifs), ainsi que des tests d'évaluation des performances. Toutes les trois ou quatre semaines, le coordinateur scientifique du district envoie aux écoles élémentaires des tests de fin de chapitre, alignés sur le rythme de progression fixé dans le guide. Notre étude porte en priorité sur ces évaluations formatives, qui comprennent cinq questions à choix multiples (dont certaines questions font l'objet d'une réponse semi-guidée) et une question ouverte. Les tests d'entraînement du district consistent quant à eux en une série de onze à plus de vingt items (les évaluations en grade 3 contiennent généralement moins d'items que celles en grade 5).

La séquence d'enseignement et d'évaluation commence par vingt-cinq jours de cours, suivis par la passation des tests d'entraînement expédiés par le coordinateur scientifique du district. La date précise du test reste à la discrétion des enseignants, mais elle précède en général de trois à cinq jours le test sommatif de fin de chapitre. Les tests d'entraînement permettent de donner des indications aux élèves ; ils sont notés mais non pris en compte dans la moyenne. Après avoir noté ces tests, puis entré et classé les informations par objectif d'apprentissage dans le système de gestion de données et remis les copies, les enseignants répartissent les élèves en plusieurs groupes selon leur performance. Dans les trois à cinq jours qui suivent ces tests, les enseignants préparent le test de fin de chapitre, qu'ils font passer aux élèves le sixième jour (soit environ 33 jours après le début du cycle). Ce test est ensuite ramassé et noté. Les enseignants envoient leurs tableaux de résultats à l'expert du programme élémentaire (elementary curriculum specialist) de l'école qui compile les résultats des élèves (par classe et par niveau), puis les transmet au directeur d'école et au coordinateur scientifique du district. Ce dernier passe alors en revue les tableaux de chaque classe avec l'expert lors de leurs réunions mensuelles. Si le besoin s'en fait sentir, l'expert rencontre (seul ou avec le coordinateur scientifique) les enseignants pour discuter des cours, de la remédiation individuelle ou de nouvelles séances de développement professionnel basées sur les résultats aux tests. 


\section{PRÉSENTATION DE LA MÉTHODOLOGIE EMPLOYÉE LORS DE CETTE ÉTUDE}

Nous nous sommes appuyée sur cinq sources de données pour mieux connaître l'usage que les enseignants faisaient des évaluations intermédiaires : les observations en classe ; les entretiens avec les enseignants; les entretiens avec les responsables de l'école et du district ; l'observation des réunions d'école et de district, ainsi que les fiches de préparation des enseignants. Chacune de ces sources de données est étudiée ci-dessous. Nous avons mené des observations en cours de mathématiques à trois reprises au cours de l'année 2006-2007 en grade 3 et grade 5. En moyenne, nous avons observé trois heures de cours par enseignant pendant l'année : une fois à l'automne, une fois en hiver et une fois au printemps. En automne, l'observation portait sur l'interaction entre l'évaluation et les cours. Plus précisément, il s'agissait d'observer les moments où les enseignants évaluaient la compréhension de leurs élèves de façon informelle et les moments où ils se servaient des résultats des évaluations intermédiaires pour adapter leur enseignement. Dans la mesure où l'objectif de l'étude était d'enquêter sur la façon dont les enseignants utilisent les informations recueillies au fil des différentes évaluations, notamment les évaluations intermédiaires, nous avons établi nos visites d'hiver et de printemps au moment où les enseignants étaient le plus susceptibles d'utiliser les informations issues de ces évaluations pour revoir un point précis du cours. II s'agissait donc de profiter d'une «fenêtre pédagogique » entre la remise des résultats de l'évaluation et la fin de cette période d'évaluation.

Comme nous n'avons pas été en mesure d'observer directement si les pratiques des enseignants étaient en rapport avec les évaluations intermédiaires, nous avons posé la question aux enseignants lors des entretiens individuels (d'une heure environ), ayant eu lieu après chaque observation de classe. Tous les entretiens furent enregistrés et retranscrits. Dans le cadre des entretiens d'automne, nous avons présenté aux enseignants un scénario d'analyse de données et une simulation de résultats d'élèves, à partir des évaluations intermédiaires de chaque district et de chaque niveau de classe. Les items de chaque scénario provenaient des évaluations intermédiaires propres à chaque district. Nous avons alors demandé aux enseignants d'imaginer qu'il s'agissait de sa classe et d'analyser les résultats à voix haute. Nous avons poursuivi (après cinq minutes de discussion maximum) par une série de questions visant à attirer l'attention de l'enseignant sur certains aspects, comme par exemple : " $Y$ a-t-il des sujets sur lesquels cette classe, dans l'ensemble, semble rencontrer des difficultés ? À quoi l'observez-vous ? "Plus précisément, nous voulions savoir si les enseignants parvenaient ou non à repérer les erreurs des élèves et ce que ces erreurs révélaient de leur réflexion, les questions qu'ils pouvaient leur poser pour vérifier leur interprétation des erreurs des élèves et les solutions pédagogiques qu'ils mettraient en œuvre pour traiter telle ou telle erreur.

Pour l'entretien d'hiver, nous avons demandé aux enseignants d'apporter des copies de leurs dernières évaluations intermédiaires et de dégager les grands traits des résultats de la classe, ainsi que les concepts mathématiques qui posaient apparemment des difficultés aux élèves. Ces questions devaient renvoyer aux questions précédentes du scénario d'analyse de données. Nous espérions ainsi avoir une image plus complète de la capacité de chaque enseignant à donner du sens aux résultats des évaluations intermédiaires. Nous avons recueilli les copies des évaluations intermédiaires en mathématiques en grade 3 et en grade 5 des élèves et nous nous sommes procuré le programme de cette matière afin de mieux comprendre les objectifs d'apprentissage à atteindre. Nous avons pu, à de nombreuses reprises, obtenir des évaluations faites par les enseignants et parfois les copies anonymes des élèves. Enfin nous avons traité tous les supports papier utilisés lors de nos observations.

La première étape du cycle d'amélioration continue consiste à recueillir des données attestant la compréhension des élèves. Les évaluations intermédiaires que nous avons analysées produisaient surtout des résultats à partir des réponses des élèves aux items des tests. Dans le district de Philadelphie, les enseignants étaient presque totalement absents de la conception des évaluations intermédiaires. À ce stade, leur mission était de se familiariser avec le système informatisé de gestion des données des tests lors des formations de formateurs dispensées par le district : il s'agissait de former une équipe restreinte par établissement qui, à son tour, devait former le reste du personnel de l'établissement. Cette formation était très limitée car elle ne concernait que de simples opérations de manipulation de l'outil informatique. En revanche, dans le district de Cumberland, les enseignants devaient noter toutes les évaluations et entrer eux-mêmes les résultats dans le système informatique du district. Même si certains ensei- 
gnants trouvaient cette opération chronophage, la plupart estimait, comme cet enseignant de grade 5 , qu'elle permettait d'observer les résultats de manière détaillée : "Maintenant c'est tout sous forme de tableaux et l'informatique, ça change tout. Ils passent le test, je les note et j'entre leurs résultats dans la base. L'ordinateur m'indique alors le pourcentage de réponses correctes. Si le pourcentage est trop faible, c'est surligné en jaune. Ça m'enlève une grosse charge de travail. Avant c'est moi qui le faisait et qui devait décider sur quels points travailler en fonction des résultats. "En outre, comme les évaluations du district de Cumberland contenaient des réponses semi-guidées et des questions ouvertes, les enseignants trouvaient utile de comparer les résultats du codage, qui permet la généralisation mais écrase les particularités individuelles, et le détail des réponses de chaque élève.

\section{ANALYSE ET INTERPRÉTATION DES RÉSULTATS OBTENUS}

Une fois les données entrées, les enseignants devaient les analyser et les interpréter pour ensuite adapter leur enseignement. L'examen des évaluations intermédiaires était une pratique courante chez les enseignants étudiés. Ils savaient tous au minimum analyser les résultats des élèves et la plupart d'entre eux accédait facilement à ces données sur informatique. Dans l'examen des données saillantes, les enseignants procédaient de la même manière. Tout d'abord, presque tous cherchaient les points faibles des élèves (pour $90 \%$ d'entre eux) et/ou passaient en revue les résultats des évaluations intermédiaires par élève et par domaine curriculaire (pour $97 \%$ d'entre eux). D'autres commençaient plutôt par regarder la performance globale de la classe (pour environ $10 \%$ des enseignants interrogés). Pour les enseignants qui cherchaient d'abord les points faibles des élèves, différentes techniques étaient utilisées, selon qu'ils regardaient directement le niveau des élèves pour tel ou tel item, ou qu'ils repéraient les résultats des élèves par discipline. Environ $20 \%$ d'entre eux commençaient par repérer les résultats des élèves faibles.

Les systèmes de gestion d'informations exerçaient une influence sur les analyses des enseignants. Dans les deux districts, ces outils permettaient de mettre en exergue les points faibles des élèves. Dans le système de gestion utilisé à Philadelphie, était relevé le pourcentage d'élèves répondant correctement à chaque item et le pourcentage d'items auxquels chaque élève répondait correctement, alors que dans celui utilisé à Cumberland, les domaines dans lesquels les élèves avaient plus d'une réponse fausse étaient surlignés en jaune. Notons toutefois que, dans les deux districts, cette influence des systèmes de gestion sur le processus d'analyse des enseignants demeurait assez limitée. À Cumberland, ce constat s'expliquait par la capacité limitée du système luimême alors qu'à Philadelphie, c'était I'utilisation moindre des fonctions plus complexes du système (telles que la production d'évaluations complémentaires), par rapport aux fonctions de base, qui était en cause.

Si l'analyse des données était pratiquée par tous les enseignants, le processus d'interprétation était beaucoup plus complexe. II dépendait de plusieurs facteurs : leurs connaissances des performances passées des élèves ou de leurs antécédents, les résultats des élèves par rapport à ceux de leurs pairs, la perception des enseignants quant à la difficulté de tel ou tel contenu mathématique, ou différents facteurs propres au district tels que la programmation des évaluations intermédiaires. À Philadelphie, l'analyse des résultats des évaluations intermédiaires a montré que les enseignants fixaient leurs propres seuils ${ }^{1}$, qui influençaient l'interprétation des données. Plus précisément, les enseignants avaient en tête un résultat minimum qui, selon eux, montrait que leurs élèves avaient acquis les concepts vus au cours des cinq semaines précédentes. Chaque enseignant fixait ses propres seuils selon les capacités de ses élèves et ses convictions quant à la difficulté des contenus et à la façon dont les élèves apprennent les mathématiques. Ces seuils variaient suivant la classe, l'élève et la période de passation du test. Ils aidaient les enseignants à fixer leurs priorités pour la semaine de révision, tant en termes de contenus à revoir que d'élèves en difficulté à cibler. En tant que tel, l'usage premier des données provenant des évaluations intermédiaires consistait apparemment à aider les enseignants à déterminer comment répartir leurs élèves en différents groupes.

L'étude a également montré que les enseignants interprétaient les résultats de leurs élèves selon leurs propres attentes, à la fois pour chaque élève et pour la classe dans son ensemble. Ils se servaient souvent des évaluations pour entériner leurs impressions quant aux forces et faiblesses de leurs élèves, impressions provenant d'évaluations précédentes, d'observations informelles et d'informations non 
scolaires. Un enseignant de grade 3 de Philadelphie témoigne : "Honnêtement, les résultats des évaluations ne sont pas une grosse surprise... On sait quand même où en sont les élèves, s'ils s'intéressent et s'ils comprennent. " Les enseignants du district de Cumberland en particulier se servaient des évaluations intermédiaires comme d'un point de départ vers davantage d'évaluations formatives. II n'était pas rare qu'ils passent en revue les problèmes de chaque élève afin de mieux savoir comment il apprend. Un enseignant de Cumberland en grade 5 témoigne : « Eh ben la première chose qu'on fait, c'est d'essayer de voir ce que fait l'élève. Sinon c'est impossible pour moi d'enseigner sans savoir comment il procède. " Ces réponses soulèvent une question plus large quant à la façon dont les enseignants prennent en charge ces nombreuses évaluations. Bien que nous ne puissions, par manque de place, détailler nos résultats, nous avons analysé comment les enseignants comparaient les différentes évaluations entre elles. Les résultats montrent que les enseignants ont recours à des types d'évaluation formative différents selon les objectifs qu'ils poursuivent. Dans notre échantillon, les évaluations intermédiaires étaient le plus souvent utilisées pour repérer des élèves en difficulté ou des contenus de programme non maîtrisés, alors que le contrôle continu (évaluations élaborées par les enseignants) servait le plus souvent à recueillir des renseignements supplémentaires sur la façon dont les élèves résolvaient les problèmes. Les évaluations élaborées par les enseignants permettaient aussi, en fonction des résultats, de modifier éventuellement leur fréquence. Tout ceci tend à montrer que les évaluations intermédiaires structurent et guident d'autres types de pratiques formatives. En ellesmêmes, elles n'éclairent guère la réflexion des élèves et leur résolution de problèmes (Goertz, Oláh \& Riggan, 2010), mais elles donnent des indications utiles aux enseignants en phase de contrôle continu.

Un des enjeux de l'analyse des résultats est d'examiner la façon dont les enseignants approfondissent cette analyse une fois qu'ils ont observé les grandes tendances des résultats de leurs classes. Afin d'enquêter sur le type de diagnostic porté par les enseignants, nous les avons tous interrogés sur les résultats des évaluations de leurs élèves et sur un petit nombre d'items de tests. Nous leur avons alors demandé ce que pouvaient bien penser leurs élèves de leurs réponses incorrectes. À nos yeux, ce moment d'analyse est un lien essentiel entre le bilan de l'évaluation et les ajustements pédagogiques nécessaires. La palette de réponses a été très large dans les deux districts. En général, ces réponses s'inscrivaient dans un continuum allant d'explications procédurales sur les erreurs des élèves à des diagnostics conceptuels. Par exemple, face à l'erreur d'un élève qui additionnait mal des fractions avec des dénominateurs différents, certains enseignants décrivaient l'incapacité de l'élève à trouver un dénominateur commun (procédure), quand d'autres faisaient remarquer que l'élève ne semblait pas avoir compris qu'un dénominateur représente le nombre de parties dans lequel le tout est divisé (concept). Dans l'ensemble, les enseignants de Philadelphie avaient plus de chances de porter un diagnostic procédural, grâce à l'organisation en réseau informatique plus complexe, que ceux de Cumberland. Par conséquent, même pour des items d'évaluation identiques, les résultats des élèves donnaient lieu à des inférences différentes de la part des enseignants.

Alors que les diagnostics initiaux des enseignants de Philadelphie s'inscrivaient plutôt dans un continuum allant du procédural au conceptuel, ceux des enseignants de Cumberland s'inscrivaient dans un continuum allant du symptomatique à l'étiologique. Par exemple, à Cumberland, concernant les erreurs des élèves sur les fractions, les enseignants apportaient des réponses symptomatiques (« Ils ont tendance à isoler soit le dénominateur, soit le numérateur») ou étiologiques («Comprennent-ils vraiment que le dénominateur sert à quelque chose ici ? "). Leurs réponses sont différentes de celles des enseignants de Philadelphie où les explications procédurales prédominent (" Ils ont additionné les deux numérateurs »). En outre, le manque d'explications ("Les élèves n'aiment pas les fractions ", "Les fractions, c'est dur pour eux ») était plus fréquent à Philadelphie qu'à Cumberland. Goertz, Oláh et Riggan (2010) font plusieurs hypothèses pour expliquer ce décalage : les enseignants de Cumberland auraient des connaissances en mathématiques plus solides, ainsi qu'un meilleur accompagnement pédagogique. Par exemple, les deux districts avaient embauché des experts en mathématiques, mais ceux de Cumberland étaient plus souvent déchargés de tout enseignement, contrairement à leurs homologues de Philadelphie, et étaient donc davantage à la disposition des enseignants, par exemple pour les accompagner dans leur interprétation des résultats des évaluations intermédiaires. Un établissement scolaire de Philadelphie disposait d'un de ces experts qui devait par ailleurs assurer tous ses cours ; en d'autres termes, il avait accepté le rôle de tuteur tout en enseignant à temps plein, ce qui limitait clairement le temps dont il disposait pour accompagner les enseignants. 
La tendance des enseignants de Cumberland à chercher la cause des erreurs de leurs élèves a amené une partie d'entre eux à s'apercevoir que leur programme de mathématiques était une source potentielle d'erreur et de mauvaise compréhension. Un enseignant de grade 5 a identifié l'utilisation d'algorithmes alternatifs et un curriculum en spirale comme des sources réelles d'erreur : "Mais c'était difficile parce qu'ils devaient utiliser la méthode des sommes partielles. Or [cet élève] aurait probablement trouvé la bonne réponse s'il avait fait une simple addition. Mais ce chapitre leur faisait faire des additions, des soustractions et des multiplications. Les sommes et produits partiels les ont donc un peu déstabilisés. " Dans le même temps, quelques enseignants ont remarqué que passer trop de temps sur un point précis du programme était également source d'erreur lors des évaluations intermédiaires. Un enseignant de grade 5 a remarqué que certains de ses élèves avaient calculé la surface d'une figure au lieu du périmètre, ce qui était demandé lors de l'évaluation. Voici son explication : «Un des problèmes de ce chapitre, c'est que la notion de périmètre est considérée comme acquise. C'est donc pas vraiment testé. Tout porte sur la surface et ça devient une habitude. C'est là où il faut leur demander s'ils ont bien lu la question. "Certes les pièges potentiels relevés par les enseignants de Cumberland et de Philadelphie ne sont pas propres à leurs programmes de mathématiques. Mais ce qui intrigue, c'est que, suite à l'analyse des résultats des évaluations intermédiaires, certains enseignants ont identifié en des termes très précis la méthode pédagogique, l'étendue du programme, les séquences de cours et les ressources curriculaires comme une source possible de mauvaise compréhension des élèves. Ce niveau de précision est important car les enseignants peuvent alors chercher des moyens également très précis de traiter les erreurs de leurs élèves, comme faire une meilleure distinction entre la méthode des produits partiels et celle des sommes partielles, ou encore donner plus d'exercices de calcul portant à la fois sur la surface et le périmètre lors du cours sur la surface.

\section{LES USAGES DES RÉSULTATS DES ÉVALUATIONS}

\section{À Philadelphie}

Alors que tous les enseignants des deux districts s'étaient engagés à faire usage des résultats des évaluations intermédiaires, à se focaliser sur les élèves aux résultats faibles et à améliorer la compréhension en mathématiques des élèves, l'étude a révélé qu'ils apportaient des réponses pédagogiques différentes à ces questions. Au cours de la semaine de révision, les enseignants de grade 3 et de grade 5 suivaient une méthode de planification pédagogique par tri : ils consacraient la plupart de leur temps et de leurs efforts aux élèves et aux parties du programme qui exigeaient le plus leur attention. Une enseignante de grade 5 explique succinctement qu'elle « ne peut pas tout revoir". À partir de leurs propres seuils fixés lors de l'analyse des résultats des items où le taux d'échec était le plus élevé, les enseignants décidaient quels élèves cibler et ce sur quoi ils devaient insister pendant les cinq jours suivant les évaluations intermédiaires. Pendant les cours, la remédiation individuelle était rare car la plupart des enseignants de Philadelphie avaient la charge d'une vingtaine d'élèves, quand ceux de Cumberland avaient non seulement moins d'élèves par classe en moyenne, mais aussi plus d'adultes à leur disposition dans chaque bâtiment scolaire pour les accompagner dans leurs pratiques. À la place, les enseignants privilégiaient le cours en classe entière, en petits groupes, et l'enseignement mutuel. Au regard des difficultés que posaient le travail en petits groupes pendant les heures de cours de mathématiques, de nombreux enseignants rencontraient les élèves par groupe en dehors des heures de classe. L'enseignement par les pairs venait en complément des cours en classe entière. En raison des ressources limitées (voir ci-dessus), nombre d'enseignants du district, livrés à euxmêmes, comptaient sur les meilleurs élèves pour aider ceux qui avaient moins bien réussi aux évaluations intermédiaires.

Quand les diagnostics des enseignants mettaient en avant des erreurs de procédure, leurs activités de remédiation portaient en priorité sur la correction de ces erreurs. Les méthodes pédagogiques ne semblaient guère dépendre du contenu enseigné ou des erreurs commises, mais plutôt de la croyance qu'une exposition à des représentations multiples du même contenu d'apprentissage est bénéfique aux élèves. Enfin un petit nombre d'enseignants donnait un test ou un quiz lors des cinq jours de remédiation, pour avoir une idée de la compréhension de leurs élèves. À Philadelphie, où les évaluations intermédiaires sont gérées selon un calendrier commun à tout le district, les enseignants faisaient remarquer qu'ils étaient nettement moins capables d'utiliser les résultats des évaluations lorsque le contenu testé n'avait pas été enseigné. Cette situation évidemment non souhai- 
table pouvait arriver lorsque les enseignants avaient pris du retard par rapport au calendrier du district ou quand ils estimaient que des items des évaluations portaient sur des contenus qui n'étaient pas censés avoir déjà été enseignés.

\section{À Cumberland}

Alors que la première initiative des enseignants de Philadelphie était de répartir leurs élèves en groupes de niveau, les enseignants de Cumberland envisageaient les évaluations intermédiaires comme un supplément d'informations pour mieux adapter la manière de répartir les élèves en groupes n'étant pas a priori des groupes de niveau. La grande majorité des cours que nous avons observés était structurée autour de cette répartition en groupes, réalisée selon les acquis et les erreurs révélés par les différentes évaluations. Si cette approche n'est guère différente de celle pratiquée à Philadelphie, elle varie au moins sur deux points. Tout d'abord, la composition des groupes ne dépend pas du niveau de performance, mais du type d'erreur faite par les élèves. Par exemple, dans un cours que nous avons observé, l'enseignant avait divisé sa classe en cinq groupes : un de ces groupes était formé d'élèves qui n'avaient pas acquis la notion de "valeur de position ", alors qu'un autre était formé d'élèves qui faisaient des erreurs de calcul récurrentes. La pédagogie de l'enseignant était dès lors ciblée sur ce type d'erreurs. En théorie, la composition des groupes pouvait évoluer, mais cette évolution ne nous a pas paru possible, dans la mesure où ces groupes étaient en réalité des groupes de niveau déguisés.

Malgré la tendance générale qu'ont les enseignants de Cumberland à analyser les résultats des évaluations intermédiaires d'après le seul système de gestion de données, leurs méthodes pédagogiques sont différentes d'un enseignant à l'autre. Certains enseignants distinguent la révision d'un concept ou d'une compétence déjà enseignée et la reprise entière d'une leçon. Cette démarche est peut-être liée au continuum interprétatif entre symptôme et étiologie : les enseignants qui comprennent l'étiologie d'une erreur savent davantage ce qu'il convient de reprendre, à quel moment et comment. Néanmoins d'autres enseignants ont une approche plus procédurale et reprennent le test d'entraînement item par item en classe entière. Enfin nous ne pouvons négliger le fait que les enseignants de Cumberland avaient un plus petit nombre d'élèves par classe et plus de supports pédagogiques à leur disposition que ceux de Philadelphie, comme du personnel supplémentaire qui les secondait lors des séances en groupes.

\section{CONCLUSION}

Presque tous les enseignants de notre échantillon ont eu recours aux évaluations intermédiaires. Ils s'en servaient surtout pour repérer les faiblesses de leurs élèves dans certaines parties du programme. Dans les deux districts, le même ensemble de facteurs influençait leur interprétation des résultats des évaluations. Parmi ces facteurs figuraient les performances antérieures de chaque élève, celles de la classe dans son ensemble et la perception de la difficulté de telle ou telle partie du programme par les enseignants. On peut également supposer que, dans ces deux districts, les supports informatiques auxquels les enseignants ont accès influencent la manière dont ils interprètent les résultats des évaluations intermédiaires. Bien que cela semble aller à l'encontre du cycle typique " recueillir, interpréter, planifier, agir ", il faut reconnaître que les enseignants savent très bien s'ils ont accès à des supports pédagogiques de qualité au moment même où ils interprètent les résultats. II est aussi possible que les enseignants disposant de moins de moyens donnent moins de sens aux données, dans la mesure où les interprétations à faire sont plus complexes. Par exemple, les enseignants de Philadelphie ne pouvaient pas prendre trois jours hors de leur temps de travail pour revoir la «valeur de position » avec les élèves, même si les résultats indiquaient que cette intention était souhaitable.

L'usage que font les enseignants des évaluations intermédiaires pose la question de savoir jusqu'à quel point ces évaluations contribuent à ce que les objectifs pour lesquels elles sont utilisées soient atteints. En d'autres termes, ces évaluations apportent-elles des informations utiles aux apprentissages des élèves en mathématiques et susceptibles d'améliorer l'enseignement ? Ces questions ont été largement ignorées dans la littérature de recherche sur l'utilisation des données issues des tests et sur les cycles d'amélioration continue de l'enseignement. Bien que cette question ne soit pas au cœur de notre étude, il nous est apparu nécessaire de développer les recherches empiriques sur la qualité des évaluations intermédiaires, dans la mesure où ces évaluations ne garan- 
tissent pas forcément l'apport d'informations diagnostiques ou pédagogiques de grande qualité (Goertz, Oláh \& Riggan, 2010). II pourrait d'ailleurs être intéressant de s'appuyer sur des dispositifs mis en œuvre par d'autres institutions éducatives aux États-Unis qui ont déjà réussi à améliorer leurs évaluations intermédiaires.

Leslie Nabors Oláh leslieno@gse.upenn.edu Graduate School of Education, Université de Pennsylvanie, États-Unis

1 Le terme « seuil " a été introduit par l'équipe de recherche : il n'était pas utilisé spontanément par les enseignants.

\section{BIBLIOGRAPHIE}

BAROODY A., FEIL Y. \& JOHNSON A. (2007). « An alternative reconceptualization of conceptual and procedural knowledge ". Journal for Research in Mathematics Education, vol. 38, n², p. 115-131.

BELL B. \& COWIE B. (2001). Formative assessment and science education. Norwell : Kluwer.

BLACK P. \& WILIAM D. (2006). " Developing a theory of formative assessment ". In J. Gardner (dir.), Assessment and learning. Londres: Sage, p. 81-100.

BULKLEY K., CHRISTMAN J., GOERTZ M. \& LAWRENCE N. (2008). " Building with benchmarks: The role of the district in Philadelphia's benchmark assessment system ». Communication présentée à l'Annual meeting of the American Educational Research Association, New York.

BURCH P. (2010). " The bigger picture: Institutional perspectives on interim assessment technologies ". Peabody Journal of Education, vol. 85, $\mathrm{n}^{\circ} 2$, p. 147162.

CLUNE W. \& WHITE P. (2008). "Policy effectiveness of interim assessments in providence public schools ". Document de travail $n^{\circ}$ 2008-10. Madison : Wisconsin Center for Education Research, University of WisconsinMadison. Disponible sur Internet à l'adresse : <http:// www.wcer.wisc.edu/publications/workingpapers/ papers.php\#W10> (consulté le 26 janvier 2011).

COBURN C. \& TALBERT J. (2006). "Conceptions of evidence-based practice in school districts: Mapping the terrain ". American Journal of Education, vol. 112, $\mathrm{n}^{\circ} 4$, p. 469-495.

CUSHMAN K. (1999). "The cycle of inquiry and action: Essential learning communities ». Old Horace, vol. 15, $n^{\circ} 4$, p. 5-17.

GOERTZ M., OLÁH L. \& RIGGAN M. (2010). From testing to teaching: The use of interim assessments in classroom instruction. CPRE Research Report RR-65. Philadelphia : Consortium for Policy Research in Education. Disponible sur Internet à l'adresse : <http:// www.cpre.org/index.php?option=com_content\&task=vi ew\&id=192\&ltemid=73> (consulté le 26 janvier 2011).
HIEBERT J. \& LEFEVRE P. (1986). « Conceptual and procedural knowledge in mathematics: An introductory analysis ». In J. Hiebert (dir.), Conceptual and procedural knowledge: The case of mathematics. Hillsdale : Lauwrence Erlbaum, p. 1-27.

KERR K., MARSH J., IKEMOTO G., DARILEK H. \& BARNEY H. (2006). " Strategies to promote data use for instructional improvement: Actions, outcomes, and lessons from three urban districts ". American Journal of Education, vol. 112, $\mathrm{n}^{\circ}$ 4, p. 496-520.

NORTHWEST REGIONAL EDUCATIONAL LABORATORY (2001). Curriculum inquiry cycle. Portland : Northwest Regional Educational Laboratory. Disponible sur Internet à l'adresse : <http://www.eric.ed.gov/PDFS/ ED426502.pdf> (consulté le 26 janvier 2011).

OLÁH L., LAWRENCE N., GOERTZ M. et al. (2007). « Testing to the test? Expectations and supports from interim assessment use ». Communication présentée à l'Annual meeting of the American Educational Research Association, Chicago.

RITTLE-JOHNSON B. \& SIEGLER R. (1998). « The relation between conceptual and procedural knowledge in learning mathematics: A review ». In C. Donlan (dir.), The development of mathematical skill. Hove : Psychology Press, p. 75-110.

RUIZ-PRIMO M. \& FURTAK E. (2004). " Informal formative assessment of students' understanding of scientific inquiry ". Document de travail CSE, Report 639. Los Angeles : University of California.

SUPOVITZ J. (2006). The case for district-based reform: Leading, building, and sustaining school improvement. Cambridge : Harvard Education Press.

TECHNOLOGY ALLIANCE (2005). The inquiry cycle. Seattle : Technology Alliance. Disponible sur Internet à l'adresse : <http://www.technology-alliance.com/ pubspols/dddm/inquirycycle.html> (consulté le 26 janvier 2011). 
TIMPERLEY H., WILSON A., BARRAR H. \& FUNG I. (2007). Teacher professional learning and development: Best evidence synthesis iteration (BES). Wellington : Ministry of Education. Disponible sur Internet à l'adresse <http://www.educationcounts.govt.nz/publications/ series/2515/15341> (consulté le 26 janvier 2011). 